\title{
Review Article \\ Preparation, Modification, and Application of Hollow Gold Nanospheres
}

\author{
Qiong-Qiong Ren, ${ }^{1}$ Ling-Yu Bai, ${ }^{1}$ Xiao-Shuai Zhang, ${ }^{1}$ Zhi-Ya Ma, ${ }^{1}$ Bo Liu, ${ }^{1}$ \\ Yuan-Di Zhao, ${ }^{1}$ and Yuan-Cheng $\mathbf{C a o}^{2}$ \\ ${ }^{1}$ Britton Chance Center for Biomedical Photonics at Wuhan National Laboratory for Optoelectronics, \\ Hubei Bioinformatics \& Molecular Imaging Key Laboratory, Department of Biomedical Engineering, College of Life Science and \\ Technology, Huazhong University of Science and Technology, Wuhan 430074, China \\ ${ }^{2}$ Key Laboratory of Optoelectronic Chemical Materials and Devices of Ministry of Education, Jianghan University, \\ Wuhan 430056, China \\ Correspondence should be addressed to Yuan-Di Zhao; zydi@mail.hust.edu.cn
}

Received 23 June 2015; Revised 24 September 2015; Accepted 7 October 2015

Academic Editor: Kimberly Hamad-Schifferli

Copyright ( 2015 Qiong-Qiong Ren et al. This is an open access article distributed under the Creative Commons Attribution License, which permits unrestricted use, distribution, and reproduction in any medium, provided the original work is properly cited.

Hollow gold nanospheres (HGNs) have great potential applications in biological sensing, biomedical imaging, photothermal therapy, and drug delivery due to their unique localized surface plasmon resonance (LSPR) feature, easy modification, good biocompatibility, and excellent photothermal conversion properties. In this review, the latest developments of HGNs in biosensing, bioimaging, photothermal therapy, and drug delivery are summarized, the synthesis methods, surface modification and bioconjugation of HGNs are also covered in this summary.

\section{Introduction}

As an important member of nanomaterials, gold nanomaterials have drawn wide attention in the biomedical field due to its numerous excellent properties $[1,2]$. There are many types of gold nanomaterials, including gold nanospheres (GNSs) [3], gold nanorods (GNDs) [4], hollow gold nanospheres (HGNs) [5], gold nanostars [6], gold nanocages [7], and gold nanorings [8]. HGNs have attracted a lot of interest due to their excellent chemical/physical properties. First, compared with gold nanospheres whose absorption peaks are located in visible region, the absorption peaks of HGNs could be adjusted to the near-infrared region $(700 \sim 900 \mathrm{~nm})$ [9]. Nearinfrared light has high penetration depth in biological tissue and fluids because these biological species lack absorbing groups in the infrared region of the spectra, which offers unparalleled advantage in photothermal treatment using near-infrared lasers. Second, HGNs have less toxicity than GNRs, because CTAB, which has biotoxicity, is not necessary in synthetic process of HGNs. In addition, hollow structure of HGNs generates minimum mass in the same size of gold nanostructures of different morphologies. The hollow structure makes them able to load medicines and other functional materials, which form significant advantages in drug delivery field. Furthermore, with a series of advantages such as good biocompatibility, excellent photothermal conversion, and easy modification with a variety of biological molecules, HGNs have broad application prospects in biomedical field. In this paper, we review the recent progress of HGNs in the fields of biomedical imaging, drug delivery, diagnostics, and photothermal therapy for disease treatments.

\section{Synthesis, Surface Modification, and Bioconjugation of HGNs}

2.1. Synthesis of HGNs. A variety of approaches have been developed for the synthesis of HGNs, mainly including sacrificial galvanic replacement of cobalt nanoparticles [10, 11], templated galvanic replacement reaction of silver for gold [12, 13], and electrochemistry [14]. In addition, there is 
another method which uses nonmetallic structures as core and gold as shell to prepare hollow gold nanospheres. Among these, template substitution has been widely used because it could adjust the SPR peak position through controlling the size of templates and the amount of chloroauric acid.

\subsubsection{Sacrificial Galvanic Replacement of Cobalt Nanoparti-} cles. Sacrificial galvanic replacement of cobalt nanoparticles is a popular method to synthetize HGNs currently. The fundamental is introduced as follows. Cobalt nanoparticles (CoPNs) with uniform size distribution are synthesized through reducing $\mathrm{CoCl}_{2}$ by $\mathrm{NaBH}_{4}$ in anaerobic environment. After $\mathrm{NaBH}_{4}$ is consumed totally, gold nanoparticles are deposited on the surface of CoPNs through replacement reaction between $\mathrm{HAuCl}_{4}$ and CoPNs. After $\mathrm{HAuCl}_{4}$ is consumed completely, unoxidized CoPNs are oxidized to $\mathrm{Co}^{2+}$ by oxygen dissolved in the solution. Sequentially, HGNs are obtained. The reaction equation is shown as follows [15]:

$$
3 \mathrm{Co}+2 \mathrm{AuCl}_{4}^{-}=2 \mathrm{Au}+3 \mathrm{Co}^{2+}+8 \mathrm{Cl}^{-}
$$

Liang et al. [15] first reported the use of sacrificial galvanic replacement of cobalt nanoparticles to synthesize uniform HGNs. They obtained HGNs in different size by adjusting the stoichiometric ratio of $\mathrm{HAuCl}_{4}$ and reducing agent $\mathrm{NaBH}_{4}$ and coated silica $\left(\mathrm{SiO}_{2}\right)$ on the surface of $\mathrm{HGNs}$, finally characterized HGNs with transmission electron microscope (TEM), high resolution transmission electron microscopy (HRTEM), scanning electron microscopy (SEM), X-ray diffraction (XRD), electron diffraction, energy-dispersive Xray analysis, and UV-visible absorption spectroscopy. The results showed that HGNs obtained by this method have uniform particle size, good dispersion, and adjustable SPR peak location. Subsequently, Schwartzberg et al. [16] also used sacrificial galvanic replacement of cobalt nanoparticles to synthesize HGNs and systematically studied the effects of various reactants to the formation of HGNs. The study showed that particle size and thickness of the shell of HGNs could be adjusted by changing the size of the cobalt template and the amount of $\mathrm{HAuCl}_{4}$. The SPR peak position of $\mathrm{HGNs}$ would be adjusted from $550 \mathrm{~nm}$ to $820 \mathrm{~nm}$ with the various ratio of HGNs particle size and shell thickness. Cobalt template size can be adjusted by changing the concentration of reducing agent $\mathrm{NaBH}_{4}$ and protective agent such as sodium citrate. The thickness of the HGNs shell can be adjusted through controlling the amount of $\mathrm{HAuCl}_{4}$ solution. HGNs with good morphology can be prepared through this method, which has been adopted by many researchers. Preciado-Flores et al. [17] made experimental improvements afterwards and they introduced polyvinylpyrrolidone (PVP) as a stabilizer to prepare HGNs with good dispersion and SPR peak in nearinfrared region $(\sim 800 \mathrm{~nm})$.

\subsubsection{Templated Galvanic Replacement Reaction of Silver for} Gold. Templated galvanic replacement reaction of silver for gold is another important method of synthetizing HGNs. The principle is that, first, silver nanoparticles (Ag NPs) are synthesized, and then gold particles deposit on the surface of silver template through replacement reaction between
$\mathrm{HAuCl}_{4}$ and $\mathrm{Ag} \mathrm{NPs}$ and gradually form gold shell. Many types of gold nanoparticles can be synthesized through this method; the morphology and size of gold nanoparticles are determined by the silver templates. Reaction equation is shown as follows [13]:

$$
3 \mathrm{Ag}_{(\mathrm{s})}+\mathrm{AuCl}_{4}^{-}(\mathrm{aq}) \longrightarrow \mathrm{Au}_{(\mathrm{s})}+3 \mathrm{Ag}_{(\mathrm{aq})}^{+}+4 \mathrm{Cl}_{(\mathrm{aq})}^{-}
$$

Zhang et al. [18] successfully prepared HGNs using Ag NPs as template. They prepared Ag NPs by reducing silver nitrate with ethylene glycol in the presence of PVP firstly and then prepared $\mathrm{HGNs}$ through replacement reaction between $\mathrm{HAuCl}_{4}$ and $\mathrm{Ag}$ NPs using $\mathrm{Ag}$ NPs as template. Prevo et al. [12] synthesized HGNs via the templated galvanic replacement reaction of silver for gold. The sizes of HGNs synthesized by this method range from 20 to $50 \mathrm{~nm}$ in diameter and the SPR absorbance of HGNs can be tuned to $800 \mathrm{~nm}$.

2.1.3. Electrochemistry. Electrochemistry is another important method of synthesizing HGNs. You et al. [19] electrodeposited silver ions on indium tin oxide (ITO) glass surface directly through cyclic voltammetry to prepare Ag NPs without any surfactant or organic ligand and then gained $\mathrm{HGNs}$ by replacement reaction between $\mathrm{HAuCl}_{4}$ and $\mathrm{Ag}$ NPs (using Ag NPs as template). HGNs surface obtained by this method can be modified with a variety of biological molecules, and the SPR peak position can be adjusted to the near-infrared region $(\sim 800 \mathrm{~nm})$, which has very important applications in the field of biosensing.

2.1.4. Other Methods. Another method of preparation HGNs is using nonmetallic structure as core and gold as shell. Liu et al. [20] synthesized hollow C-60 nanometer shell, firstly. Then using the hollow C-60 nanometer shell as template, gold particles were deposited on the surface of C-60 by electrochemical reduction. Finally HGNs were deposited. Graf and van Blaaderen [21] synthesized $\mathrm{SiO}_{2}$ nanoparticles by Stöber method firstly and adsorbed gold particles on the surface to form gold shell and finally obtained hollow gold nanospheres by dissolving $\mathrm{SiO}_{2}$ core with hydrofluoric acid. Zhong et al. [22] used the cross-linked product of glucose oxidase (GOD) and glutaraldehyde as template and then used ascorbic acid to reduce $\mathrm{HAuCl}_{4}$ and adsorbed gold particles on the template surface and finally prepared gold nanospheres with hollow structure.

2.2. Surface Modification and Bioconjugation of HGNS. In order to enhance the biocompatibility or achieve particular function of HGNs, it is necessary to modify the surface. HGNs have good surface chemical property, which can bind a lot of small organic molecules or biological macromolecules together in covalent or noncovalent manner.

In order to reduce electrostatic and hydrophobic interactions between nanoparticles and avoid being cleared by human reticuloendothelial system (RES), biocompatible stabilizers are often modified on the surface of nanoparticles. Polyethylene glycol (PEG) is a commonly used stabilizer, which can effectively prevent the nanoparticles being cleared 
by the RES, and thus the nanoparticles have longer cycle time in the body [23]. In addition to PEG [19], there are many materials for surface modification of HGNs, such as silicon dioxide [15], PVP [17], dextran [24], sodium citrate [25], and the likes.

Drugs or nanoparticles generally have two approaches going into the tumor site: passive targeting and active targeting. Passive targeting aggregates drugs or nanoparticles at the tumor site through enhanced permeability and retention effect (EPR effect) of solid tumor tissue. However, this targeting approach is not effective for all tumors, because the degree of tumor angiogenesis and polarity will be different due to different tumor types and states. In addition, passive targeting is also easy to induce multidrug-resistant, which is not conducive to the treatment of tumors [9]. Active targeting makes up the deficiency of passive targeting. In active targeting, nanoparticles and the targeted molecules are bound in some ways. Directional marker to tumor is achieved through the bond between targeted molecules and tumor-specific cell surface receptor and ultimately attains treatment purposes. Active targeting can enrich nanoparticles or drugs at the tumor site initiatively which could significantly improve the accuracy of treatment and effectively reduce the digestion of drugs in body. Therefore, active targeting has significant advantages in treatment of tumors.

Commonly used tumor targeted molecules include antibody molecules [26], polypeptide [27], aptamer [28], antagonist [29], and folic acid (FA) [30]. Liu et al. [31] applied HGNs conjugated with monoclonal antibody (anti-TROP 2 ) to photothermal therapy of cervical cancer (HeLa) cells. The monoclonal antibodies can specifically bind highly expressed trophoblast cell surface antigen 2 (TROP2) of HeLa cells. Studies have showed that under the laser irradiation of right intensity, comparing with tumor cells incubated with nontargeted probes, the growth of tumor cells incubated with HGNs that conjugated monoclonal antibodies was significantly inhibited. Tian et al. [27] compared the intake behaviors between HGNs probes with targeted RGD (arginine-glycineaspartic acid) peptide IA-RGD-PEG-HGNs and probes without targeted molecules after the intravenous injection in mouse liver cancer model. The results showed that, $24 \mathrm{~h}$ after intravenous injection, intake of probes with RGD was much higher than that without RGD $(0.20$ versus $0.099 \% \mathrm{ID} / \mathrm{g}$; $P<0.001$ ), which showed advantages of active targeting compared with passive targeting.

\section{Biomedical Applications of HGNs}

HGNs are widely used in biosensing, biomedical imaging, photothermal cancer therapy, and delivery transportation of drugs or genes.

3.1. HGNs in Biological Sensing. Gold nanoparticles (Au NPs) have unique localized surface plasmon resonance (LSPR) characteristic. The LSPR peak position is closely linked with morphology, size, and surrounding medium of nanoparticles, especially the refractive index (RI) of matter close to the nanoparticle surface. Compared with solid gold nanospheres (SGNs), HGNs have a higher refractive index sensitivity
$[32,33]$. LSPR sensors based on changing the LSPR peak position of Au NPs have been widely used in biosensing [34, 35]. Liu et al. [36] modified HGNs to the surface of electrode using crosslinking agent 1, 6-thiol, and fixed DNA on the modified electrode to prepare novel electrochemical sensors for DNA. They studied hybridization between the probe and the target DNA by cyclic voltammetry and differential pulse voltammetry. The results showed that HGNs can significantly enhance the hybridizing ability of DNA. The DNA sensor had lower detection limit ( $1 \mathrm{pM})$, wide dynamic range (1 pM $10 \mathrm{nM}$ ), high stability, and reusability. Zhang et al. [37] fabricated an electrochemiluminescence (ECL) biosensor based on HGNs modified graphene (C60-rGO) and glucose oxidase (GOX), which could be used to detect knife legumin A (ConA). GOX could catalyze the oxidation of glucose and in situ react with a large number of hydrogen peroxides. HGNs would catalyze ECL reactions between luminol and hydrogen peroxide. The joint action of GOX and HGNs caused ECL luminol signal strength to be greatly enhanced. The biosensor had high sensitivity, which could detect ConA within $0.10 \sim 100 \mathrm{ng} / \mathrm{mL}$ and the detection limit was as low as $30 \mathrm{pg} / \mathrm{mL}(\mathrm{SNR}=3)$. These findings explained the important applications of HGNs in nanobiosensing aspect.

3.2. Biomedical Imaging of HGNs. The biomedical imaging applications of HGNs include transmission electron microscopy (TEM), computed tomography (CT), surface-enhanced Raman scattering (SERS) imaging, and photoacoustic imaging (PAI). Among these imaging modes, PAI has drawn much attention. Photoacoustic imaging is a noninvasive biomedical imaging method developed in recent years. Biological tissue generates heat under laser irradiation. The heat results in local temperature increases and thus leads to thermal expansion and pressure wave which means photoacoustic signals. Through the information of body optical absorption characteristics carried in photoacoustic signals, researchers can reconstruct the image of the body optical absorption distribution [38]. Combining the dual advantages of deep penetration in ultrasound imaging and high selectivity in optical imaging, tissue photoacoustic imaging with high contrast and high resolution can be obtained. The most important advantage of photoacoustic imaging is that photoacoustic signal phase and amplitude of sample can be measured directly without pretreatment. The operation is simple and could maintain the natural state of biological sample, which can be detected in vivo.

HGNs are excellent photoacoustic contrast agents, because they can effectively enhance the specific absorption of organizations and have much higher photoacoustic efficiency than blood. Lu et al. [39] intravenously injected the PEG-modified HGNs (PEG-HGNs) in nude mice. Two $h$ later, the photoacoustic image was displayed. Photoacoustic image obtained using PEG-HGNs as contrast agent had high contrast resolution, in which the diameter of vessels was as thin as $100 \mu \mathrm{m}$, and the contrast agent had no obvious toxicity to the liver, spleen, and kidneys of nude mice. The results showed that the PEG-HGNs was a very promising contrast agent in photoacoustic imaging and had high 
resolution and sensitivity. Lee et al. [40] monitored the temperature of tumor site using optoacoustic imaging during the photothermal therapy. The results showed that tumor temperature was raised from $37^{\circ} \mathrm{C}$ to over $50^{\circ} \mathrm{C}$ when shining a laser on to the tumor injected with DOX@PEG-HGNs probes. It showed the promising applications of HGNs for photoacoustic imaging and monitoring the temperature of the tumor.

3.3. HGNs in Photothermal Therapy of Tumors. Cancer is a serious threat to the health of people around the world. Traditional cancer treatments (such as surgery, chemotherapy, and radiation therapy) have significant limitations. For example, surgery and radiation therapy cannot solve the problem of systemic metastasis of tumor cells; the chemotherapy needs to send drugs to all parts of the body through blood vessels, so the body cells, whether malignant cells, are damaged, which causes a lot of side effects. Photothermal therapy has become a new cancer therapy. The basic principle is that changing the environment of tumor cells by the method of laser irradiation results in the tumor tissue temperature being raised to the unbearable temperature $\left(41 \sim 47^{\circ} \mathrm{C}\right)$ [41]. Then, different sensitivity of normal cells and tumor cells to temperature results in necrocytosis and achieves the purpose of treatment of cancer, finally. Photothermal therapy attracts extensive attention because the advantages of less damaging normal tissue and their immune system. Near-infrared light has a good tissue penetration in the human body because human tissues and body fluids have few absorption of light in the near-infrared region. Photothermal therapy using nearinfrared light as the light source has been widely used in the treatment of tumors.

At present, scientists have developed a number of nanometer materials for photothermal therapy of cancer, such as carbon nanotubes [42], gold nanometer materials [43], and copper sulfide [44], which can convert light energy into heat under laser irradiation and finally kill cancer cells. Currently, the research on photothermal conversion materials focuses on the gold nanometer materials.

In the gold nanomaterials with different morphologies and structure, HGNs exhibit unparalleled advantages in photothermal therapy of tumor because of so many advantages such as spherical shape, small size, absorption peak which can be adjusted to the near-infrared region, easily modified with a variety of biological targeted molecules on the surface, and good biocompatibility. Conjugating biological targeted molecules (antibodies or ligands) on HGNs and binding with targeted molecules (antigens or receptors) on the surface of tumor cells result in HGNs concentrating in the tumor cells. Then near-infrared light is irradiated to tumor cells. HGNs absorb light and convert light energy into heat energy. It is a very attractive photothermal therapy model to kill tumor cells [45].

Lu et al. [46] prepared targeted biological probes of NDP-MSH-PEG-HGNs, which could actively enriched in melanoma cells of mouse, by coupling targeted molecules of $\alpha$-melanocyte stimulating hormone analogue (NDP-MSH) with HGNs and modifying stabilizer of PEG. They compared the survival condition of cells being incubated with the targeted probes and nontargeted probes under the same laser irradiation conditions. The results showed that the targeted probes had maximum lethality to cells under near-infrared laser irradiation, while nontargeted probes, independent laser irradiation, or targeted probes had small lethality. Melancon et al. [47] conjugated antibody anti-EGF to the HGNs to prepare anti-EGFR-HGNs probes which could be positioned in the A431 cells of epidermal carcinoma cell line. The probes were applied to the photothermal therapy at cellular level. The results showed that the majority of A431 cells incubated with targeted probes were killed, while cells under laser irradiation alone or incubated with nonspecific targeted probe under laser irradiation almost had no damage. The results proved the targeted HGNs have excellent effect on photothermal therapy of tumor cells. Thus, the HGNs conjugated with targeted molecules have a very important practical value in the near-infrared photothermal therapy of tumor.

3.4. HGNs in Drug Delivery. Chemotherapy is an important method of treating cancer. Many anticancer drugs are difficult to give full play to efficacy in treatment because of poor stability, low solubility, being nontargeted, and easy removal by metabolism. However, loading cancer drugs on a suitable support cannot only extend residence time of drugs in the body, thereby improving the utilization of drugs, but also control the release of drugs. In addition, if the carrier is conjugated with targeted molecules, it can also greatly reduce the cytotoxicity of drugs to normal cells caused by no targeting. Ideal drug carriers should have characteristics of chemically stable, long half-life, nontoxic or low toxic, biodegradable, large drug loading capacity and others. Among the many nanometer materials, HGNs have been widely used as drug carriers because of cavity structure, large specific surface area, good biological safety and biocompatibility, and easy surface functional modification. You et al. [48] loaded the antitumor drug doxorubicin (DOX) with GNSs and HGNs (shell thickness of $4 \mathrm{~nm}$ ) of same gold content and size of $40 \mathrm{~nm}$. If DOX can only be loaded on the surface, drug load of HGNs will be twice that of GNSs, theoretically. However, the results showed that drug load on HGNs was 3.5 times that of GNSs. Thus, the drug was not only present in the outer surface of HGNs but also loaded in their cavity, which made drug loading capacity of HGNs greatly increased. Zhao et al. [49] coupled antitumor drug DOX and biological targeted molecule aptamer (Apt) to HGNs and studied the releasing process of DOX at low $\mathrm{pH}$. The results showed that, at $\mathrm{pH}$ 5.0 or 6.0 , DOX released $80 \%$ and $68 \%$ in $2 \mathrm{~h}$, respectively, while, at $\mathrm{pH} 7.4$, DOX released only $7.5 \%$ within $2 \mathrm{~h}$. The normal human blood $\mathrm{pH}$ is 7.35 7.45, and $\mathrm{pH}$ of early and late endosomes in tumor cells is 6.0 and 5.0, respectively. Therefore, drug loading system of HGNs has a significant advantage in reducing the side effects of drugs.

In addition to transport drugs, HGNs can also be used to transport genes. Lu et al. [50] coupled small interfering RNA (siRNA) and biological targeted molecules folic acid (FA) to HGNs. HGNs delivered siRNA to tumor cells. Under 
the near-infrared laser irradiation, siRNA separated from $\mathrm{HGN}$ and bound the transcription factor NF- $\kappa \mathrm{B}$ (the transcription factor plays a role in genetic expression of tumor cells), which made it not able to function properly and thereby inhibited tumor cell growth.

3.5. HGNs in Combined Photothermal Therapy and Chemotherapy. Near-infrared (NIR) laser induced photothermal therapy (PTT) is considered a good choice to treat tumor cells; however, it is difficult to eradicate tumor cells by PTT alone because of the nonuniform heat distribution. There have been quite a few studies that combine PTT with chemotherapy and the results are proved to be better than PTT or chemotherapy alone. You et al. [51] loaded antitumor drug DOX to PEG coated hollow gold nanospheres (DOX@PEGHAuNS); they studied the antitumor activity of DOX@PEGHAuNS combined with NIR laser in vitro and in vivo. They found that the nanoparticles displayed decreased systemic toxicity compared to free DOX or liposomal DOX and exhibited enhanced antitumor effect after the irradiation of laser. You et al. [52] also synthesized DOX-loaded hollow gold nanospheres that target EphB4 (T-DOX@HAuNS). They confirmed the release of DOX from DOX@HAuNS after being treated with NIR laser by dual radiotracer technique and proved the greater tumor growth inhibiting effect of TDOX@HAuNS plus laser compared to HAuNS plus laser.

\section{Conclusion}

HGNs plays an important role in biosensing field, especially in LSPR sensors. Meanwhile, its applications in biomedical field have drawn much more attention. Diagnosis and treatment drugs of combining imaging and treatment in nanostructures have received an astonishing amount of attention in the field of cancer diagnosis and treatment. Compared with other types of gold nanostructure, HGNs have many advantages such as small size, hollow spherical structure, and strong absorption peak which can be adjusted to nearinfrared region. In particular, its cavity structure can be used to load functional reagent (like anticancer drugs) and then achieve the combination of light therapy and chemotherapy, which has important applications in anticancer medicine. Besides, the conjugation of HGNs to biomolecular can target for the tumors and make it more effective to treat tumors. It can be predicted that multifunctional nanoparticles based on HGNs will play an increasingly important role in the integrated treatment of tumors.

\section{Conflict of Interests}

The authors declare that there is no conflict of interests regarding the publication of this paper.

\section{Authors' Contribution}

Qiong-Qiong Ren and Ling-Yu Bai equally contributed to this paper.

\section{Acknowledgments}

This work was supported by the National Natural Science Foundation of China (Grant nos. 81271616 and 81471697), the Foundation for Innovative Research Groups of the NNSFC (Grant no. 61121004), the Natural Science Foundation of Hubei Province (2014CFB1010), the Key Technology R\&D Program of Hubei Province (2014BBB003), and Yellow Crane Talent (Science \& Technology) Program of Wuhan City.

\section{References}

[1] E. H. Jeong, G. Jung, C. A. Hong, and H. Lee, "Gold nanoparticle (AuNP)-based drug delivery and molecular imaging for biomedical applications," Archives of Pharmacal Research, vol. 37, no. 1, pp. 53-59, 2014.

[2] S. Alex and A. Tiwari, "Functionalized gold nanoparticles: synthesis, properties and applications-a review," Journal of Nanoscience and Nanotechnology, vol. 15, no. 3, pp. 1869-1894, 2015.

[3] M. Brust, M. Walker, D. Bethell, D. J. Schiffrin, and R. Whyman, "Synthesis of thiol-derivatised gold nanoparticles in a twophase liquid-liquid system," Journal of the Chemical Society, Chemical Communications, no. 7, pp. 801-802, 1994.

[4] Y.-Y. Yu, S.-S. Chang, C.-L. Lee, and C. R. C. Wang, "Gold nanorods: electrochemical synthesis and optical properties," Journal of Physical Chemistry B, vol. 101, no. 34, pp. 6661-6664, 1997.

[5] M. Chandra, A.-M. Dowgiallo, and K. L. Knappenberger Jr., "Controlled plasmon resonance properties of hollow gold nanosphere aggregates," Journal of the American Chemical Society, vol. 132, no. 44, pp. 15782-15789, 2010.

[6] Q. L. Cui, B. H. Xia, S. Mitzscherling et al., "Preparation of gold nanostars and their study in selective catalytic reactions," Colloids and Surfaces A: Physicochemical and Engineering Aspects, vol. 465, pp. 20-25, 2015.

[7] S. E. Skrabalak, J. Y. Chen, Y. G. Sun et al., "Gold nanocages: synthesis, properties, and applications," Accounts of Chemical Research, vol. 41, no. 12, pp. 1587-1595, 2008.

[8] T.-T. Chi, Y.-C. Tu, M.-J. Li et al., "Photothermal optical coherence tomography based on the localized surface plasmon resonance of Au nanoring," Optics Express, vol. 22, no. 10, pp. 11754-11769, 2014.

[9] K. J. Cho, X. Wang, S. M. Nie, Z. Chen, and D. M. Shin, "Therapeutic nanoparticles for drug delivery in cancer," Clinical Cancer Research, vol. 14, no. 5, pp. 1310-1316, 2008.

[10] S. Adams, D. Thai, X. Mascona, A. M. Schwartzberg, and J. Z. Zhang, "Key factors affecting the reproducibility of synthesis and growth mechanism of near-infrared absorbing hollow gold nanospheres," Chemistry of Materials, vol. 26, no. 23, pp. 68056810, 2014.

[11] R. Campardelli, G. Della Porta, L. Gomez, S. Irusta, E. Reverchon, and J. Santamaria, "Au-PLA nanocomposites for photothermally controlled drug delivery," Journal of Materials Chemistry B, vol. 2, no. 4, pp. 409-417, 2014.

[12] B. G. Prevo, S. A. Esakoff, A. Mikhailovsky, and J. A. Zasadzinski, "Scalable routes to gold nanoshells with tunable sizes and response to near-infrared pulsed-laser irradiation," Small, vol. 4, no. 8, pp. 1183-1195, 2008.

[13] Y. G. Sun, B. T. Mayers, and Y. N. Xia, "Template-engaged replacement reaction: a one-step approach to the large-scale 
synthesis of metal nanostructures with hollow interiors," Nano Letters, vol. 2, no. 5, pp. 481-485, 2002.

[14] T. T. Hu, Y. Y. Lin, J. L. Yan, and J. W. Di, "Synthesis of hollow gold nanoparticles on the surface of indium tin oxide glass and their application for plasmonic biosensor," Spectrochimica Acta A: Molecular and Biomolecular Spectroscopy, vol. 110, pp. 72-77, 2013.

[15] H.-P. Liang, L.-J. Wan, C.-L. Bai, and L. Jiang, "Gold hollow nanospheres: tunable surface plasmon resonance controlled by interior-cavity sizes," Journal of Physical Chemistry B, vol. 109, no. 16, pp. 7795-7800, 2005.

[16] A. M. Schwartzberg, T. Y. Olson, C. E. Talley, and J. Z. Zhang, "Synthesis, characterization, and tunable optical properties of hollow gold nanospheres," The Journal of Physical Chemistry B, vol. 110, no. 40, pp. 19935-19944, 2006.

[17] S. Preciado-Flores, D. Wang, D. A. Wheeler et al., "Highly reproducible synthesis of hollow gold nanospheres with near infrared surface plasmon absorption using PVP as stabilizing agent," Journal of Materials Chemistry, vol. 21, no. 7, pp. 23442350, 2011.

[18] Z.-S. Zhang, Z.-J. Yang, X.-L. Liu, M. Li, and L. Zhou, "Multiple plasmon resonances of $\mathrm{Au} / \mathrm{Ag}$ alloyed hollow nanoshells," Scripta Materialia, vol. 63, no. 12, pp. 1193-1196, 2010.

[19] J. You, J. L. Zhou, M. Zhou et al., "Pharmacokinetics, clearance, and biosafety of polyethylene glycol-coated hollow gold nanospheres," Particle and Fibre Toxicology, vol. 11, no. 1, article 26, 2014.

[20] C. Liu, W. H. Dong, M. Liu, Y. Fan, and L. Zhen, "Preparation and study of C-60 hollow nanoshells," Acta Chimica Sinica, vol. 67, no. 16, pp. 1825-1828, 2009.

[21] C. Graf and A. van Blaaderen, "Metallodielectric colloidal coreshell particles for photonic applications," Langmuir, vol. 18, no. 2, pp. 524-534, 2002.

[22] X. Zhong, Y.-Q. Chai, and R. Yuan, "A novel strategy for synthesis of hollow gold nanosphere and its application in electrogenerated chemiluminescence glucose biosensor," Talanta, vol. 128, pp. 9-14, 2014.

[23] G. Prencipe, S. M. Tabakman, K. Welsher et al., "PEG branched polymer for functionalization of nanomaterials with ultralong blood circulation," Journal of the American Chemical Society, vol. 131, no. 13, pp. 4783-4787, 2009.

[24] Y. T. Lim, M. Y. Cho, B. S. Choi, Y.-W. Noh, and B. H. Chung, "Diagnosis and therapy of macrophage cells using dextrancoated near-infrared responsive hollow-type gold nanoparticles," Nanotechnology, vol. 19, no. 37, pp. 375105-375112, 2008.

[25] E. Hao, S. Li, R. C. Bailey, S. Zou, G. C. Schatz, and J. T. Hupp, "Optical properties of metal nanoshells," Journal of Physical Chemistry B, vol. 108, no. 4, pp. 1224-1229, 2004.

[26] X. Yang, Y. Liang, J. Liu, Z. L. Chen, and X. S. Yang, "Anti-cmet antibody conjugated hollow gold nanospheres as a new nanomaterial for enhancing the effect of photothermal ablation therapy," International Journal of Gynecological Cancer, vol. 24, no. 9, pp. 646-647, 2014.

[27] M. Tian, W. Lu, R. Zhang et al., "Tumor uptake of hollow gold nanospheres after intravenous and intra-arterial injection: PET/CT study in a rabbit VX2 liver cancer model," Molecular Imaging and Biology, vol. 15, no. 5, pp. 614-624, 2013.

[28] M. P. Melancon, M. Zhou, R. Zhang et al., "Selective uptake and imaging of aptamer- and antibody-conjugated hollow nanospheres targeted to epidermal growth factor receptors overexpressed in head and neck cancer," ACS Nano, vol. 8, no. 5, pp. 4530-4538, 2014.
[29] W. Lu, C. Xiong, R. Zhang et al., "Receptor-mediated transcytosis: a mechanism for active extravascular transport of nanoparticles in solid tumors," Journal of Controlled Release, vol. 161, no. 3, pp. 959-966, 2012.

[30] S. Barbosa, A. Topete, M. Alatorre-Meda et al., "Targeted combinatorial therapy using gold nanostars as theranostic platforms," The Journal of Physical Chemistry C, vol. 118, no. 45, pp. 26313-26323, 2014.

[31] T. Liu, J. G. Tian, Z. L. Chen et al., "Anti-TROP2 conjugated hollow gold nanospheres as a novel nanostructure for targeted photothermal destruction of cervical cancer cells," Nanotechnology, vol. 25, no. 34, Article ID 345103, pp. 103-114, 2014.

[32] J. Z. Zhang and C. Noguez, "Plasmonic optical properties and applications of metal nanostructures," Plasmonics, vol. 3, no. 4, pp. 127-150, 2008.

[33] M. M. Miller and A. A. Lazarides, "Sensitivity of metal nanoparticle surface plasmon resonance to the dielectric environment," Journal of Physical Chemistry B, vol. 109, no. 46, pp. 21556-21565, 2005.

[34] B. Sepúlveda, P. C. Angelomé, L. M. Lechuga, and L. M. LizMarzán, "LSPR-based nanobiosensors," Nano Today, vol. 4, no. 3, pp. 244-251, 2009.

[35] M. E. Stewart, C. R. Anderton, L. B. Thompson et al., "Nanostructured plasmonic sensors," Chemical Reviews, vol. 108, no. 2, pp. 494-521, 2008.

[36] S. F. Liu, J. Liu, X. P. Han, Y. N. Cui, and W. Wang, "Electrochemical DNA biosensor fabrication with hollow gold nanospheres modified electrode and its enhancement in DNA immobilization and hybridization," Biosensors \& Bioelectronics, vol. 25, no. 7, pp. 1640-1645, 2010.

[37] J. J. Zhang, Y. Ruo, S. H. Chen, X. Zhong, and X. P. Wu, "A sandwich-like electrochemiluminescent biosensor for the detection of concanavalin $\mathrm{A}$ based on a $\mathrm{C}_{60}$-reduced graphene oxide nanocomposite and glucose oxidase functionalized hollow gold nanospheres," RSC Advances, vol. 4, no. 89, pp. 4846548471, 2014.

[38] H. F. Zhang, K. Maslov, G. Stoica, and L. V. Wang, "Functional photoacoustic microscopy for high-resolution and noninvasive in vivo imaging," Nature Biotechnology, vol. 24, no. 7, pp. 848851, 2006.

[39] W. Lu, Q. Huang, G. Ku et al., "Photoacoustic imaging of living mouse brain vasculature using hollow gold nanospheres," Biomaterials, vol. 31, no. 9, pp. 2617-2626, 2010.

[40] H. J. Lee, Y. Liu, J. Zhao et al., "In vitro and in vivo mapping of drug release after laser ablation thermal therapy with doxorubicin-loaded hollow gold nanoshells using fluorescence and photoacoustic imaging," Journal of Controlled Release, vol. 172, no. 1, pp. 152-158, 2013.

[41] L. O. Svaasand, C. J. Gomer, and E. Morinelli, "On the physical rationale of laser induced hyperthermia," Lasers in Medical Science, vol. 5, no. 2, pp. 121-128, 1990.

[42] X. J. Wang, C. Wang, L. Cheng, S.-T. Lee, and Z. Liu, "Noble metal coated single-walled carbon nanotubes for applications in surface enhanced raman scattering imaging and photothermal therapy," Journal of the American Chemical Society, vol. 134, no. 17, pp. 7414-7422, 2012.

[43] H.-X. Xia, X.-Q. Yang, J.-T. Song et al., "Folic acid-conjugated silica-coated gold nanorods and quantum dots for dualmodality CT and fluorescence imaging and photothermal therapy," Journal of Materials Chemistry B, vol. 2, no. 14, pp. 1945-1953, 2014. 
[44] Q. W. Tian, M. H. Tang, Y. G. Sun et al., "Hydrophilic flowerlike cus superstructures as an efficient $980 \mathrm{~nm}$ laser-driven photothermal agent for ablation of cancer cells," Advanced Materials, vol. 23, no. 31, pp. 3542-3547, 2011.

[45] J. Z. Zhang, "Biomedical applications of shape-controlled plasmonic nanostructures: a case study of hollow gold nanospheres for photothermal ablation therapy of cancer," Journal of Physical Chemistry Letters, vol. 1, no. 4, pp. 686-695, 2010.

[46] W. Lu, C. Y. Xiong, G. D. Zhang et al., "Targeted photothermal ablation of murine melanomas with melanocyte-stimulating hormone analog-conjugated hollow gold nanospheres," Clinical Cancer Research, vol. 15, no. 3, pp. 876-886, 2009.

[47] M. P. Melancon, W. Lu, Z. Yang et al., "In vitro and in vivo targeting of hollow gold nanoshells directed at epidermal growth factor receptor for photothermal ablation therapy," Molecular Cancer Therapeutics, vol. 7, no. 6, pp. 1730-1739, 2008.

[48] J. You, G. Zhang, and C. Li, "Exceptionally high payload of doxorubicin in hollow gold nanospheres for near-infrared lighttriggered drug release," ACS Nano, vol. 4, no. 2, pp. 1033-1041, 2010.

[49] N. X. Zhao, J. You, Z. H. Zeng, C. Li, and Y. L. Zu, "An ultra $\mathrm{pH}$-sensitive and aptamer-equipped nanoscale drug-delivery system for selective killing of tumor cells," Small, vol. 9, no. 20, pp. 3477-3484, 2013.

[50] W. Lu, G. Zhang, R. Zhang et al., "Tumor site-specific silencing of NF- $\kappa$ B p65 by targeted hollow gold nanosphere-mediated photothermal transfection," Cancer Research, vol. 70, no. 8, pp. 3177-3188, 2010.

[51] J. You, R. Zhang, G. D. Zhang et al., "Photothermal-chemotherapy with doxorubicin-loaded hollow gold nanospheres: a platform for near-infrared light-trigged drug release," Journal of Controlled Release, vol. 158, no. 2, pp. 319-328, 2012.

[52] J. You, R. Zhang, C. Y. Xiong et al., "Effective photothermal chemotherapy using doxorubicin-loaded gold nanospheres that target EphB4 receptors in tumors," Cancer Research, vol. 72, no. 18, pp. 4777-4786, 2012. 

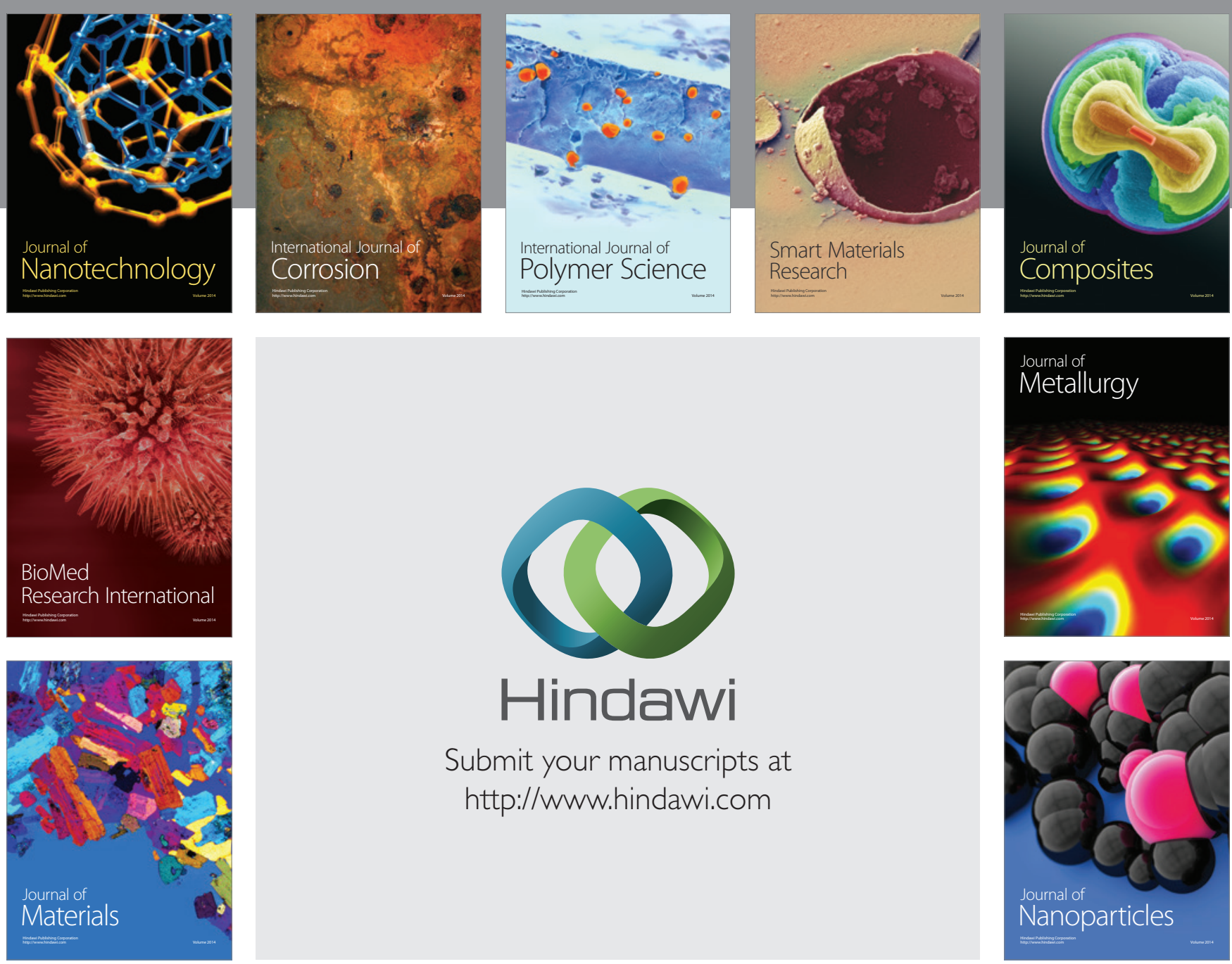

Submit your manuscripts at http://www.hindawi.com
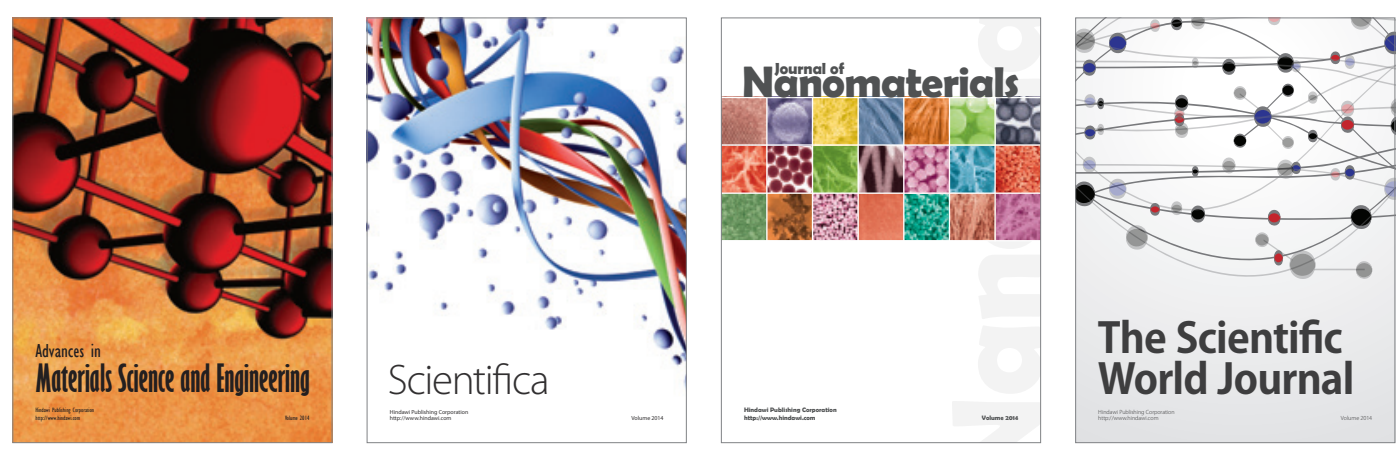

\section{The Scientific World Journal}
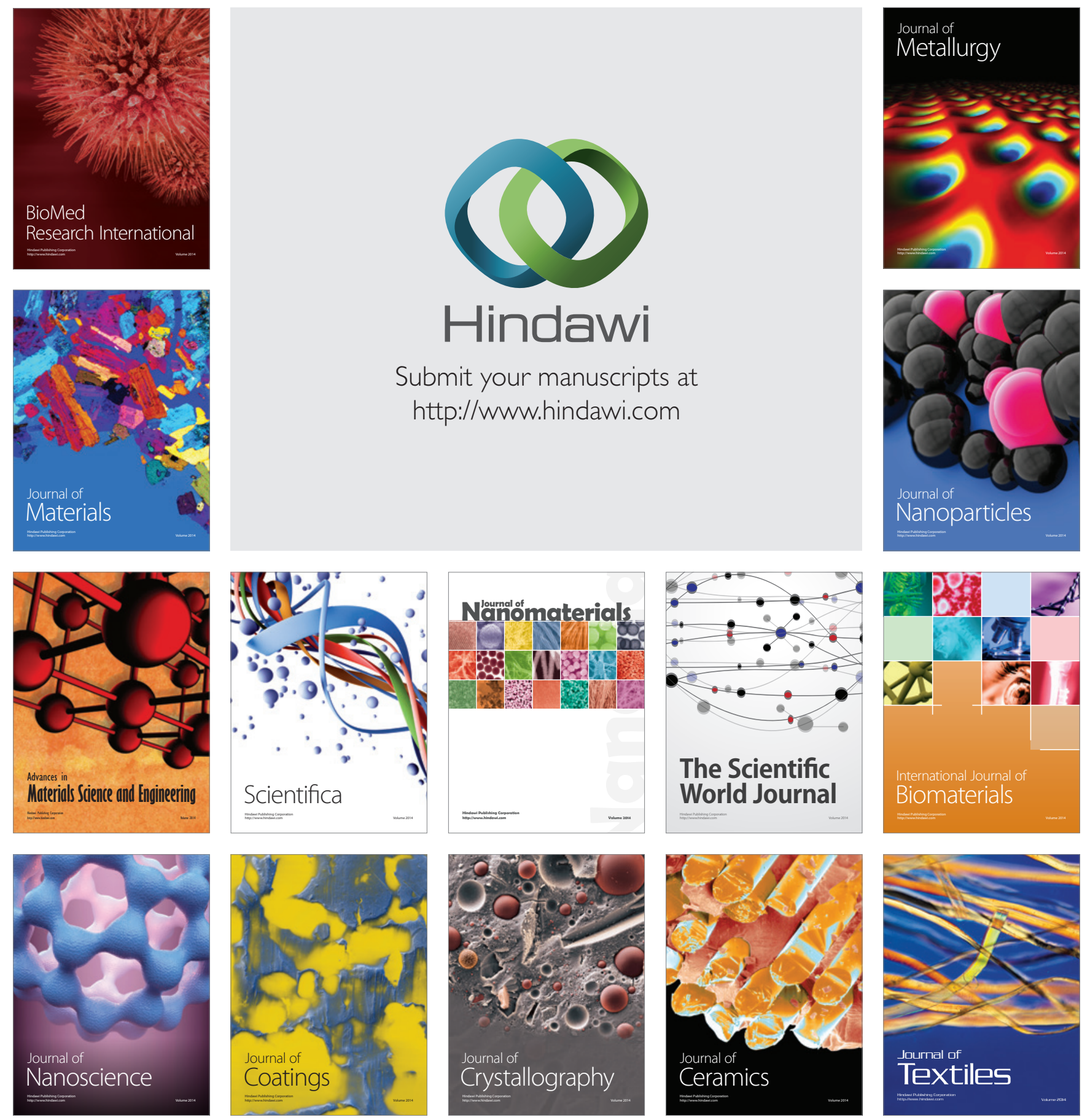